\title{
¿Qué hay que decir a nuestros pacientes sobre el cigarrillo electrónico?
}

El uso de los cigarrillos electrónicos ha crecido de forma significativa en los últimos años, y se espera que supere en USA a los ingresos de la venta de cigarrillos en unos 10 años. Se promocionan como más saludables, y como una ayuda potencial para dejar de fumar, con la ventaja de poder fumar ("vapear") en cualquier lugar. Sus comercializadores, significativamente entre ellos las potentes compañías tabaqueras, nos dicen que son "más saludables, más limpios y más baratos".

El crecimiento de su uso está relacionado, en gran medida, con el hecho de que cuestan menos que los cigarrillos al no tener impuestos específicos, y sabemos que al menos uno de cada cinco fumadores los ha probado. Sin embargo, hay serias dudas sobre su seguridad y su utilidad, y son objeto de revisiones científicas recientes como la publicada en la revista Circulation ${ }^{1}$ de la American Heart Asociation, o la de nuestro compañero Rodrigo Córdoba en la revista Atención Primaria ${ }^{2}$. Por otra parte, diversas sociedades científicas como el Comité Nacional de Prevención del Tabaquismo (CNPT) ${ }^{3}$ o la semFYC ${ }^{4}$ se han posicionado sobre ellos.

Seguramente ya algún paciente nos ha preguntado sobre estos dispositivos en la consulta, y nos puede ser útil conocer unas pequeñas orientaciones para responder a sus preguntas o dudas.

\section{Utilidad para dejar de fumar}

Los cigarrillos electrónicos podrían ser una ayuda para dejar de fumar, porque combinan el uso de nicotina (sustancia adictiva que justifica gran parte de la adicción al tabaco) y los aspectos conductuales y psicológicos del fumar. Además, parece que los fumadores los aceptan con más facilidad que el uso de la terapia sustitutiva con nicotina, ya que mimetizan mejor el acto de fumar. Se calcula que la mitad de los fumadores que han intentado dejar de fumar han usado estos dispositivos, siendo el $20 \%$ del total de fumadores el porcentaje de los que los han probado. Aunque existen serias dudas sobre su utilidad como una ayuda para dejar de fumar, los médicos de familia tenemos que estar receptivos cuando nos pregunten los pacientes sobre su utilidad, ya que nos están enviando un mensaje indirecto de que quieren o se están planteando dejar de fumar. Esto nos da una oportunidad de intervenir como profesionales sanitarios sobre el consumo de tabaco.
Cada "calada" de cigarrillo electrónico, con el más alto contenido de nicotina, tiene solo un $20 \%$ de la nicotina de una bocanada de un cigarrillo, por lo que son menos potentes, y no ofrecen el mismo nivel de nicotina al que está acostumbrado el fumador. Esto hace que sean menos placenteros y no cumplan con las expectativas de los fumadores que los usan.

Hay que decir que la eficacia de los cigarrillos electrónicos para ayudar a las personas a dejar de fumar no se ha demostrado científicamente. No hay suficientes estudios que demuestren que los cigarrillos electrónicos pueden ser una ayuda efectiva y la mayoría de las afirmaciones que se hacen son inexactas, engañosas o están insuficientemente probadas. Se trata generalmente de estudios de pequeña envergadura, con limitaciones metodológicas claras y/o conflictos de intereses por parte de sus autores. Un estudio reciente realizado en Nueva Zelanda ha comparado la eficacia para ayudar a dejar de fumar de los cigarrillos electrónicos con los parches de nicotina, y estos fueron moderadamente eficaces para ayudar a los fumadores a dejar de fumar, con un logro similar de abstinencia. Los autores concluyen que se necesita más investigación con urgencia para establecer claramente sus beneficios generales y los daños a nivel individual y poblacional. También hay que comentar que no se ha comparado su eficacia con otros fármacos que han demostrado su utilidad para dejar de fumar como el bupropion o la vareniclina.

Algunos fumadores los usan al mismo tiempo que los cigarrillos convencionales (uso dual) para poder seguir fumando en lugares cerrados. Este uso dual puede diferir el abandono del tabaco y puede llevar a un consumo excesivo de nicotina.

\section{Seguridad de uso}

Es obvio que los cigarrillos electrónicos son menos nocivos que los cigarrillos convencionales, pero esto no quiere decir que sean inocuos. Debido a la falta de regulación y controles de calidad, la composición puede variar mucho entre diferentes marcas y dentro de una misma marca, y pueden contener dosis detectables de otros tóxicos (nitrosaminas, metales pesados, etc.) que no aparecen en el etiquetado, como han demostrado estudios de la Agencia de Medicamentos y Alimentos (FDA) 
de EEUU. Además, contienen propilenglicol, glicerina, disolventes, aromatizantes, conservantes y nicotina, que es una sustancia adictiva, tóxica y con efectos sobre el sistema cardiovascular.

Algunos estudios han mostrado efectos fisiológicos adversos a corto plazo del vapor que desprenden sobre la función pulmonar de voluntarios sanos, sin que sea posible establecer claramente la relevancia clínica, y sin poder documentar por ahora los efectos de la exposición continuada, ni los efectos a medio y largo plazo. También se han documentado intoxicaciones por nicotina por ingesta de recambios de los cigarrillos electrónicos.

Uno de los aspectos no resueltos del cigarrillo electrónico es su posible utilidad dentro de una estrategia de reducción de daños. Al usar cigarrillos electrónicos en vez de cigarrillos con tabaco se podría evitar la inhalación de sustancias tóxicas como el alquitrán y otras. Esta visión del uso del cigarrillo electrónico para disminuir el daño cuenta con sus defensores dentro de la profesión sanitaria, como la organización británica 'Action on Smoking and Health' que los contempla como una estrategia de reducción de daños ${ }^{5}$. Hay que tener en cuenta que la evidencia para promocionar el tabaco sin humo como estrategia de salud pública es débil e inconsistente.

\section{¿Qué dicen las autoridades sanitarias?}

La Organización Mundial de la Salud (OMS) nos informaba en julio de 2013 que se desconoce si el uso de los cigarrillos electrónicos puede ayudar a vencer la adicción o por el contrario puede ayudar a mantenerla o iniciarla entre los menores. Nos recor- daba que no hay evidencia científica consistente para apoyar el uso terapéutico de estos productos y de manera contundente concluye así: "Hasta que no haya datos, certificados por un organismo regulador nacional competente, que demuestren que los cigarrillos electrónicos son productos seguros, eficaces y de calidad aceptable, se debería advertir seriamente a los consumidores que se abstengan de utilizarlos".

Recientemente, la OMS ha dado a conocer un nuevo informe (en agosto de 2014) sobre el uso y regulación de los cigarrillos electrónicos, en el que pide a los países miembros mayores medidas regulatorias sobre estos dispositivos y que se prohíba la venta de cigarrillos electrónicos a los menores, estimando que su consumo es "una grave amenaza" para ellos. El documento aconseja la prohibición del consumo en espacios públicos cerrados "al menos hasta que se pruebe que el vapor que producen no es peligroso para las personas que están alrededor"7.

\section{¿Qué hay que decir a los pacientes?}

Para aquel paciente que nunca ha fumado la respuesta cuando nos pregunten sobre su uso es simple: NO los use. Si nos preguntan nuestros pacientes fumadores sobre el uso de estos dispositivos, nuestra respuesta debe moverse entre la cautela y la precaución (tabla 1), y aprovechar la pregunta para intervenir sobre el consumo de tabaco con estrategias que han demostrado su eficacia. Una combinación de fármacos y consejos que se han demostrado efectivos para dejar de fumar es la mejor opción ${ }^{8}$.

- Probablemente los cigarrillos electrónicos causen menos daños en la salud que los cigarrillos convencionales.

- No está demostrado que ayuden a dejar de fumar. Si los está usando ahora, le aconsejo que establezca una fecha de abandono del tabaco.

- Si usted quiere dejar de fumar se le puede ayudar con una combinación de fármacos y consejos que se han demostrado efectivos.

- No es aconsejable su uso al mismo tiempo que los cigarrillos convencionales (uso dual).

- Su uso como estrategia de reducción de daños del fumar no está demostrada.

- Los cigarrillos electrónicos tiene menos sustancias dañinas, lo cual no quiere decir que sean inocuos. El vapor de agua que desprenden podría afectar a la salud. No vapee en sitios cerrados y delante de otras personas.

Tabla 1. Qué responder si nos preguntan sobre los cigarrillos electrónicos 


\section{Bibliografía}

1. Bhatnagar A, Whitsel LP, RibisI KM, Bullen C, Chaloupka F, Piano MR, Robertson RM, McAuley T, Goff D, BenowitzN. MD Electronic Cigarettes: A Policy Statement From the American Heart Association. Circulation. August 24, 2014. Disponible en: http://circ.ahajournals.org/content/early/2014/08/22/ CIR.0000000000000107.full.pdf [consultado el 10/09/2014]

2. Córdoba García R. El desafío de los cigarrillos electrónicos. Aten Primaria. 2014;46(6):307-312. Disponible en: http:// zl.elsevier.es/es/revista/atencin-primaria-27/articulo/el-desafiolos-cigarrillos-electronicos-90332040?referer=buscador [consultado el 10/09/2014]

3. Comité Nacional de Prevención del Tabaquismo. Informe sobre los cigarrillos electrónicos. Madrid. Diciembre 2013. Disponible en: http://www.cnpt.es/documentacion/publicaciones/4a0 615f922e444ab5117990c3ede662e5ad64f098dfec2909e0fd3abed374295.pdf consultado el 10/09/2014]

4. Ribera Osca JA, Córdoba García R y Gascó García P. Miembros del Grupo de Abordaje del Tabaquismo (GAT) de la semFYC. El cigarrillo electrónico: la utopia del cigarrillo seguro. Aten Primaria. 2014;46(2):53-54. Disponible en: http://apps.elsevier.es/watermark/ctl_servlet?_f=10\&pident articulo $=90268990$ \&pident usuario $=0$ \&pcontactid $=\&$ pident revi

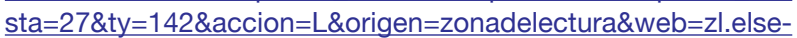
vier.es\&lan $=e s \& f i c h e r o=27 v 46 n 02 a 90268990 p d f 001$.pdf [consultado el 15/09/2014].
5. Action on Smoking and Health. Electronic cigarette. 2013. Disponible en: http://ash.org.uk/files/documents/ASH_715.pdf [consultado el 15/09/2014].

6. WHO. Questions and answers on electronic cigarettes or electronic nicotine delivery systems (ENDS), Statement. WHO. Geneva, 9 July 2013. Disponible en: http://www.who.int/tobac$\mathrm{co/communications/statements/eletronic} \mathrm{cigarettes/en/} \mathrm{[con-}$ sultado el 15/09/2014].

7. Sistemas electrónicos de administración de nicotina Informe de la OMS. Conferencia de las Partes en el Convenio Marco de la OMS para el Control del Tabaco. Julio 2014. Disponible en http://apps.who.int/gb/fctc/PDF/cop6/FCTC_COP6_10-sp. pdf?ua $=1$ [consultado el 15/09/2014].

8. Camarelles F, Mataix J, Cabezas C, Lozano J, Ortega G et al. Guía para el tratamiento del tabaquismo activo y pasivo. BarceIona 2011. Sociedad Española de Medicina Familiar y Comunitaria. http://www.semanasinhumo.es/upload/file/Guia bolsiIlo_tabaquismo_2011_2(1).pdf [consultado el 15/09/2014].

\section{Francisco Camarelles Guillem}

Médico de Familia. Grupo de Abordaje al Tabaquismo de la semFYC y Grupo de Educación Sanitaria y Promoción de la Salud del PAPPS 